\title{
PEX1 and PEX7 Genes are Necessary for Completion of Immune Pathways and Survival Post-Infection
}

Sara Murphy

Dalhousie University

ABSTRACT: The innate immune system consists of a variety of different immune pathways including the c-Fos and NF-KB immune pathways and is the first line of an organism's defence against infection. This research analyzes the role of peroxisomes, an organelle present in eukaryotic cells, in the regulation of the c-Fos mediated pathway in innate immunity. Quantitative PCR was used to measure how reduced peroxisome function affected the relative expression of c-Fos in human fibroblasts. The effects of impaired peroxisome function on the overall ability of an organism to fight off infection were investigated by tracking Drosophila melanogaster survival post-infection with $E$. coli. In addition, the effects of peroxisomes on NF-KB regulated pathway were quantified in $D$. melanogaster fibroblasts by measuring the relative expression of NF-KB. Results showed that at least partial peroxisome function is necessary for the c-Fos and NF- KB mediated pathways and that without peroxisomes, $D$. melanogaster has a significantly reduced chance of survival post-infection. Interestingly, greater concentrations of fatty acids in the cell were shown to have an inhibitory effect on the cell's ability to complete the immune response. Overall, this study provides evidence that peroxisomes are necessary for innate immune function. More extensive future research on peroxisome function could be used to applied to better understand and treat immune disorders.

KEYWORDS: Peroxisomes, Immunity, Phagocytosis, Drosophila melanogaster

\section{INTRODUCTION}

\section{The Immune System and Response}

The innate immune system is present in all organisms and is essential to their survival. It allows organisms to eliminate microbial threats using a series of processes. The cellular immune response begins with macrophages in mucosal tissues, which form the first line of defence against the environment. Macrophages are found in all animals and are responsible for sensing pathogens and beginning the immune response [1]. Macrophage signalling brings more blood cells to the infected area and thereby, recruiting additional phagocytes.

Phagocytes are important in the inflammatory immune response, travelling throughout the circulatory system searching for any possible threats. When a pathogen is detected and assessed using phagocytic receptors, it is engulfed into the cell. Once inside the cell, the pathogen is engulfed by a phagosome. The pathogen is killed by reactive oxygen species or proteolytic enzymes there [2].
Along with macrophages, fibroblasts are another important tool that can be used to study the immune response. Fibroblasts are the main cell type present in connective tissue. This tissue is what creates physical barriers, such as skin, which defends against infection. Fibroblasts are the first cells to recognize invading pathogens. They then react by secreting cytokines which warn other cells of the pathogen and activates effector cells to destroy the pathogen. Fibroblasts also produce antimicrobial peptides that help fight off the pathogen [3]. Whereas macrophages circulate the body in search of invading pathogens, fibroblasts fight off the invasion by simply changing its secretions.

\section{The Peroxisome}

The peroxisome is an organelle found in the cytoplasm of all eukaryotic cells that is suspected to be important to the immune response. In animal cells, peroxisomes are responsible for the degradation or biosynthesis of several molecules essential to the cell's function [4]. They are 
responsible for the synthesis of plasmalogens, an important molecule in the immune system. Another important function is to protect cells from degradation due to reactive oxygen species $[5,6]$. Peroxisomes also contain over fifty enzymes that are essential for digestion and have a diverse array of functions.

Peroxins are proteins that maintain the function of peroxisomes. There are over thirty peroxins responsible for various functions encoded by peroxisomal biogenesis factor (PEX) genes. The PEX1 gene encodes for a protein responsible for mediating preperoxisomal vesicles in the formation of peroxisomes and recycling AAA proteins. The PEX7 gene encodes for a cargo protein and acts as a receptor for molecules being shuttled to the peroxisome [6].

\section{Molecules Important to Immune Pathways}

Other important molecules to the immune response are NF-KB and c-Fos. C-Fos is a family of four genes responsible for cell proliferation and differentiation [7]. It is also involved in regulating immuno-genes and can be used as an indicator of immune activity [5]. NF-KB is a family of protein complexes that induce the expression of inflammatory genes, leading to increased cytokine production. It also regulates the activation of inflammatory $\mathrm{T}$ cells. Following an infection, NF-KB activates a series of responses in the cell by inducing the canonical and non-canonical NF-KB pathways, which use different signalling methods to stimulate the inflammatory response. This ultimately leads to NF-KB being concentrated in the nucleus of the cell for gene transcription. This can be used as an indicator that the cell has undergone the full canonical or non-canonical NF-KB pathways $[8,9]$.

In addition, palmitate, or palmitic acid, is a lipid synthesized by peroxisomes that is important in the immune response. Palmitate helps to stimulate the inflammatory response by increasing the production of pro-inflammatory genes, activating a macrophage into a pro-inflammatory state. Palmitate rapidly increases the transcription of proinflammatory genes while also reducing transcription factors for anti-inflammatory genes [10].

\section{Objectives}

This research builds on previous studies that suggested peroxisomes have a role in innate immunity. Prior research by Di Cara [5] showed that when peroxisome function was compromised by eliminating the PEX5 and PEX7 genes, GFP-
SKL S2 Drosophila melanogaster (D. melanogaster) cells were no longer able to perform phagocytosis. Immunofluorescence data showed that while control GFP-SKL S2 cells phagocytosed latex beads, the mutant cells could not [5]. When infected with bacteria, the PEX deficient cells phagocytosed fewer bacteria than their control counterparts. This was evident by the lack of vacuoles being formed in the cytoplasm of the cell. In addition, it was shown that PEX deficient $D$. melanogaster were more susceptible to infection and that PEX deficient cells phagocytosed fewer bacteria than wild-type cells [5].

This research tests new possible roles of the peroxisome in immunity by analyzing the effects of reduced peroxisome functionality in several aspects of the innate immune system. First, we determined that PEX genes have a role in the immune signalling undergoing in a cell since PEX1 deficient fibroblasts showed a reduction in the expression of c-Fos. Secondly, the ability of $D$. melanogaster deficient in PEX7 to survive after being infected with Escherichia coli (E. coli) was greatly reduced when compared to control wild-type $D$. melanogaster. Finally, the immune pathways of NF-KB were analyzed using a D. melanogaster macrophage with a knockdown in the PEX7 gene and showed that the cells were unable to complete the immune response.

\section{METHODOLOGY}

\section{PEX genes and the c-Fos Mediated Immune Pathway}

First, human fibroblasts were collected from patients affected with peroxisome biogenesis disorders. Fibroblasts can be collected by performing a skin biopsy and isolating only the dermis and epidermis [11]. Fibroblasts with a deficiency in the PEX1 gene and fibroblasts with a deficiency in the PEX7 gene were compared to a control human fibroblast using qPCR analysis. Fibroblast cells were cultured using Dulbecco's Modified Eagle Media (DMEM) and 10\% fetal bovine serum at $37{ }^{\circ} \mathrm{C}$ at $5 \% \mathrm{CO}_{2}$ concentration. Cells were cultured for approximately one week before working with them and were sub-cultured every 5-7 days. Cells were then harvested using trypsin treatment to detach the cells from adhering to container walls. Ribonucleic acid (RNA) was extracted using a Qiagen RNeasy kit then quantified using Nanodrop technology at a wavelength of $260 \mathrm{~nm}$. The RNA was then used to make complementary deoxyribonucleic acid (cDNA). This was then used in a real-time quantitative polymerase chain reaction (PCR) to measure the expression level of c-Fos. This measures 
the c-Fos mediated immune signalling that is occurring in the cell. Glyceraldehyde 3-phosphate dehydrogenase (GAPDH) was used as an internal control to compare the relative change of c-Fos. Since GAPDH is a housekeeping gene, its activity is constant despite changes to the cell. Allowing us to measure the relative change of c-Fos in comparison [12]. $\Delta \Delta$ cycle threshold (CT) calculations were used to measure the significance of the results, as well as standard deviation calculations to measure error. These calculations measure the expression level of the target gene. The data was then compared using the relative fold change.

\section{Eliminating the PEX1 gene in D. melanogaster}

Transcription of the PEX1 gene was reduced using small interfering RNA (siRNA) interference creating a knockdown for that gene. In this technique, the siRNA recognizes the specific PEX1 gene. Then a dicer complex is added which splices the RNA. This reduces the amount of gene products made by the $P E X$ gene, diminishing its functionality.

\section{Infection of D. Melanogaster}

The survival rates of PEX7 deficient $D$. melanogaster were measured following infection with $E$. coli. A HML-GAL4 strain was crossed with UAS PEX7 to create the genotype $H M L$ GAL4 $\propto$ UAS PEX7. First generation D. melanogaster were then placed in a vial containing mashed banana infected with approximately $100 \mu \mathrm{g}$ of $E$. coli. The adult $D$. melanogaster were kept in an incubator at $25^{\circ} \mathrm{C}$ and counted every two days to determine the number of remaining flies. The survivorship of infected control $D$. melanogaster was recorded as well as noninfected PEX7 deficient D. melanogaster. Results were then analyzed statistically using a logrank test J on Prism statistics.

\section{PEX1 and the NF-KB Pathway}

D. melanogaster SR2+ macrophages from a cell line were infected with $10 \mu \mathrm{L} E$. coli and incubated for three hours at $37^{\circ} \mathrm{C}$. Six different macrophages were created for this section; one treated with $10 \mu \mathrm{L}$ palmitate prior to infection, one that was simply infected, and one uninfected macrophage all with a deficiency in the PEX1 gene. The same three conditions were repeated using control macrophages as well. RNA was extracted by adding $100 \mu \mathrm{L}$ of chloroform then using a Qiagen RNeasy kit. The macrophages then underwent a realtime quantitative PCR reaction using NF-KB as the target messenger RNA (mRNA). NF-KB replication indicates that
NF-KB went to the nucleus of the cell and the full immune response was completed. $\Delta \Delta C T$ calculations will be done to determine the expression level of NF-KB. The relative fold change and standard deviation calculations were used to compare the different groups.

\section{RESULTS}

\section{C-Fos Expression in PEX1 and PEX7 Deficient Fibroblasts}

The intent of this test was to determine if the PEX1 and PEX7 genes have an effect on the c-Fos immune pathway. Figure 1 shows the $\Delta \Delta C T$ values, representing the relative fold change of the expression of c-Fos, for a control fibroblast, a PEX1 deficient fibroblast (PEX1 null), and a PEX7 deficient fibroblast (PEX7 null). PEX1 null shows a statistically significant difference in c-Fos expression from controls, whereas PEX7 does not (Fig.1).

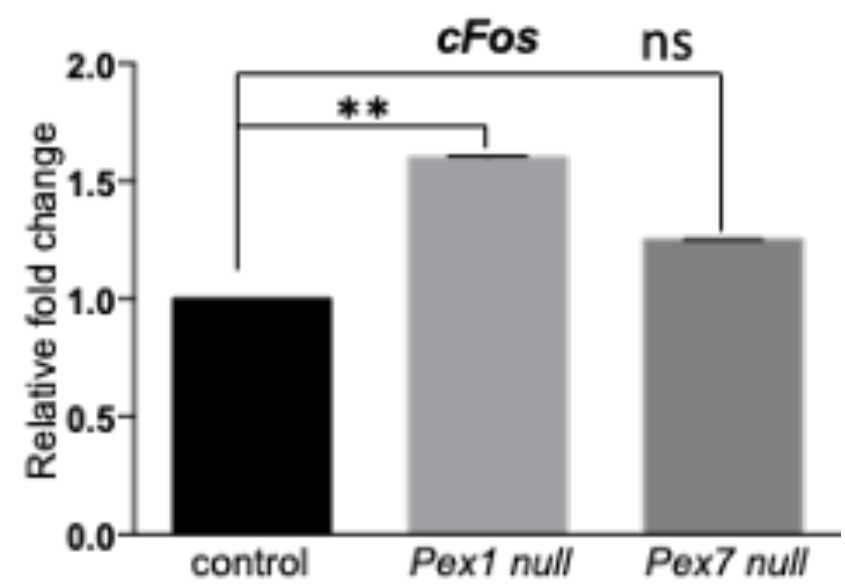

Figure 1. Bar graph comparing the $\triangle \Delta C T$ values of c-Fos expression in wild-type control, PEX1 null, and PEX7 null human fibroblasts. ns indicates not significant. Double asterisks indicate a $p$ value of $<0.01$.

\section{Survival of PEX1 Deficient Flies Post-Infection}

The purpose of this test was to observe if $D$. melanogaster can survive infection without functional peroxisomes. The survivorship curve of $D$. melanogaster post oral infection with E. coli is shown in Figure 2. This curve represents the percent of survivors left after every two days. Observations were carried out for a total of eight days. This shows that PEX1 null D. melanogaster died sooner than the PEX1 wild-type controls. After only six days, PEX1 null $D$. melanogaster were completely eliminated while about $60 \%$ of control D. melanogaster 
remained alive. They also died sooner-after only two days- whereas control $D$. melanogaster began dying after four days (Fig. 2).

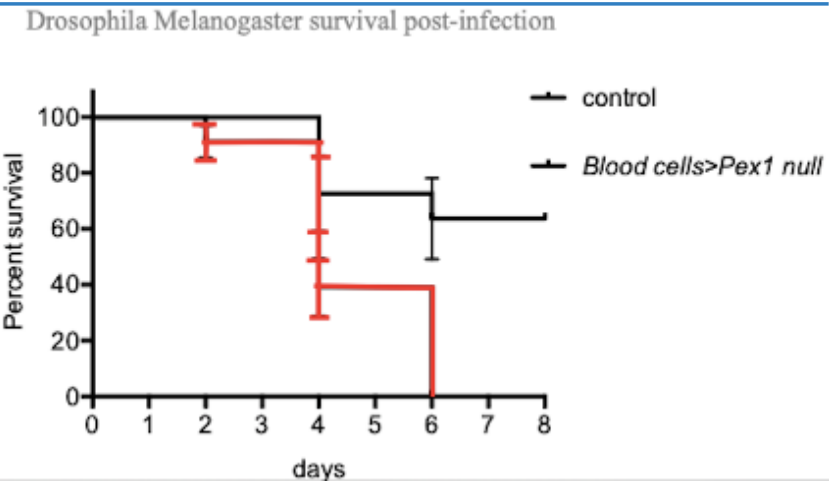

Figure 2. Survivorship curve showing the percent of living control D. melanogaster and PEX1 null D. melanogaster. The sample size of $D$. melanogaster was 15.

\section{The Effect of the PEX1 Gene and Palmitate on the Expression of NF-KB}

For the final test, the goal was to determine if the PEX1 gene has an effect on the NF-KB immune pathway. Figure 3 shows the $\triangle \Delta C T$ values for NF-KB expression in control $D$. melanogaster macrophages and PEX1 null macrophages; without being infected, following infection with $E$. coli, and following treatment with palmitate and infection. The results show a significant difference in the expression of NF-KB following infection of the control cell. There was little expression of NF-KB after palmitate treatment in control cells. Mutant cells show little difference in expression of c-Fos under all conditions. The only macrophages showing a significant change in NF-KB expression from the uninfected control were the infected control macrophages which had a significance level of $p<0.0001$ (Fig. 3).

\section{DISCUSSION}

Results from the qPCR test on human fibroblasts show that PEX1 null fibroblasts have a significant reduction in the expression level of c-Fos compared to wild-type cells, whereas PEX7 null did not. This shows the PEX1 gene is necessary for the c-Fos immune pathway whereas PEX7 is not. However, PEX7 could still have a role in a different immune pathway, so it may still be of importance to the immune system. These results could be due to PEX1 having a broader role in peroxisome function. PEX1 has multiple different functions

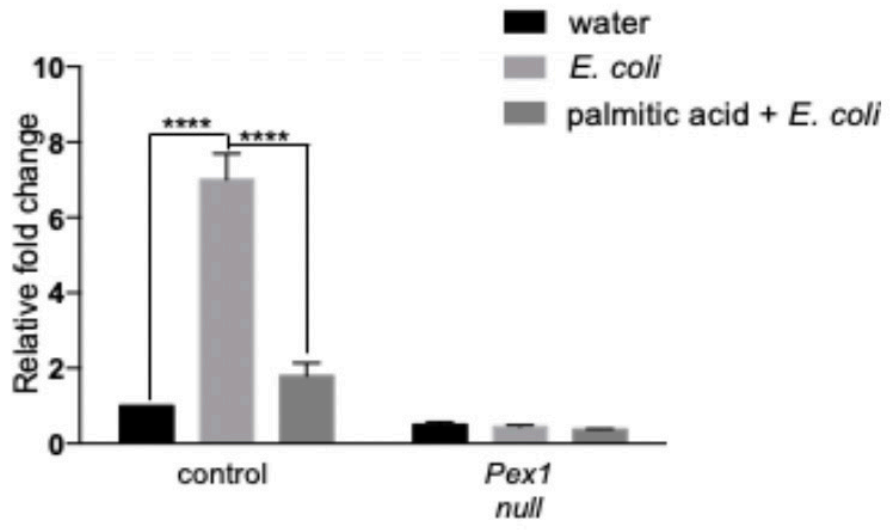

Figure 3. $\triangle \triangle \mathrm{CT}$ values for NF-KB expression in control $D$. melanogaster macrophages and PEX1 null macrophages. Macrophages are uninfected, infected with $E$. coli, or treated with $10 \mu \mathrm{L}$ of palmitate then infected. Quadruple asterisks indicate a $p$ value of $<0.0001$.

in the peroxisome and is more important in the general function of peroxisomes. The PEX1 genes have been shown to be necessary for peroxisome assembly and lack of this gene can cause various tissue defects in embryos as well as defects in neuron function and organization. The majority of peroxisome biogenesis disorders are caused by mutations in the PEX1 gene [13]. PEX7 is much more specialized as its main role is in recognizing and transporting PTS2 cargo. It also aids in regulating the size of peroxisomes [14]. Since the peroxisome is not completely debilitated without the PEX7 gene, the cell is still able to complete the c-Fos regulated immune response.

Data from the survivorship of $E$. coli infected $D$. melanogaster shows that $P E X 1$ null $D$. melanogaster are unable to survive post-infection. All PEX1 deficient $D$. melanogaster were unable to survive six days after infection, whereas about $60 \%$ of control $D$. melanogaster survived. This suggests that the innate immune system is impaired without the function of peroxisomes. These results support data from the first gene expression analysis on human fibroblasts, which demonstrated that PEX1 null human fibroblasts had a significant change in the expression level of c-Fos. The survivorship curve suggests that peroxisomes are necessary for a number of immune pathways and are necessary for organisms to survive an infection. In the immune response, there are many different immune pathways and for the PEX1 gene to have such a drastic effect on the organism's survival, it must affect the majority of these pathways. 
Finally, results from the gene expression analysis of NF$\mathrm{KB}$ in $D$. melanogaster macrophages results showed that only infected control macrophages had a significant change in the expression level of NF-KB compared to uninfected control macrophages. This is the normal reaction in a cell following infection and is to be expected [8]. However, the infected control treated with palmitate showed a much-reduced expression level of NF-KB in comparison with the untreated cell. Palmitate is a fatty acid known to stimulate the inflammatory response [15]. However, data from the qPCR suggests that a greater than normal concentration of fatty acids can impair the immune response [16]. Previous research has shown that while fatty acids stimulate the production of T-cells in lower concentrations, fatty acids can reach a toxic concentration where they induce apoptosis in T-cells. Apoptosis of T-cells could be what is inhibiting the immune response [16]. PEX1 null D. melanogaster macrophages showed little change in the expression of NF-KB after infection. This shows that the peroxisome is necessary to complete the NF-KB immune pathway. This data supports the results of the c-Fos expression in human fibroblasts and survivorship of infected D. melanogaster, which both concluded that PEX1 is important in innate immunity and offers evidence that at least two - c-Fos and NF-KB, immune pathways are downregulated by impairment of peroxisomes. These results are also supported by immunofluorescence data by Di Cara (Fig. S1). Data shows that in control D. melanogaster cells relish, an NF-KB homolog, enters the nucleus of the cell. In PEX1 null cells, relish surrounds the nucleus but does not enter. This suggests that the immune pathway cannot be completed without the use of peroxisomes.

Overall, this data supports previous research by Di Cara [5], which determined that $D$. melanogaster deficient in PEX5 and PEX7 were unable to survive post-infection with $E$. coli. Our research supports this by demonstrating that PEX1 is also necessary for survival post-infection. In addition, past research has shown that peroxisomes are necessary to induce the AMP immune pathway [5]. Our research suggests that peroxisomes are necessary for NF-KB regulated immune pathways as well as c-Fos mediated pathways. This adds to the theory that peroxisomes could be necessary for the innate immune system as a whole, not only in a single pathway.

This research had a number of limitations encountered along the way. The $D$. melanogaster survivorship curve was originally going to include PEX7 deficient $D$. melanogaster as well however difficulties in working with $D$. melanogaster limited our abilities to include them. It would be interesting to measure the rate of survival of PEX7 deficient flies to see if their survival supports data from the analysis of PEX7 concerning the c-Fos immune pathway. While data showed PEX7 did not affect the c-Fos pathway, testing survival rates could indicate if the gene is affecting the immune system in a different way. In addition, the second QPCR reaction was originally going to be immunofluorescence testing. In this case, both are different representations of the same data. The goal of this test was to measure if the NF-KB pathways were being completed. The pathways end with the localization of NF-KB in the nucleus of the cell and the expression of NF-KB [8]. Immunofluorescence data would have shown if the NF-KB had reached the nucleus, whereas QPCR measured if NF-KB was being expressed. In future studies, it would be beneficial to use both immunofluorescence and QPCR to measure what part of the NF-kB pathways was stopped. This could be done by measuring multiple important molecules in the canonical and non-canonical NF-KB pathways, such as IKKa or IKK $\beta$, using the two methods. With further research, this could indicate exactly how peroxisomes affect the NF-KB pathways.

The next step in research would be to determine exactly how peroxisomes are affecting the innate immune system. Our research showed that peroxisomes are necessary for both the NF-KB immune pathway and the c-Fos immune pathway. This shows that peroxisomes play a role in cell signalling and cell proliferation following infection. Future research should address how peroxisomes are affecting these processes and analyze other immune pathways as well. It would be interesting to analyze pathways in the adaptive immune system such as the APRIL pathway or the interferon pathway. It would also be beneficial to study pathways involved in cancer, such as the p53-mediated apoptosis pathway, to see if peroxisome deficiencies have any relation to cancerous tumour growth.

An interesting area for future study would be to analyze if peroxisomes have a role in phagocytosis. Peroxisomes appear to have an effect on the entire innate immune system and target the system as a whole rather than individual sections. Phagocytosis is necessary for all immune pathways in targeting pathogens and bringing them into the cell. Past research by Di Cara [5] showed that the PEX5 and PEX7 genes were necessary for phagocytosis. It can be theorized that peroxisomes are necessary for phagocytosis and without 
them the cell is unable to take in pathogens, resulting in reduced immune function.

In the future, it would be beneficial to analyze a murine model as they have a larger genome and could provide some variance in the effects concerning the knockdown of PEX genes. A murine model more closely resembles humans and could provide more insight into how peroxisomes affect our own immune systems. This research has the potential to provide more information about our own immune system as well as peroxisome biogenesis disorders.

With future research, this information could be used to create new treatments or medicines. Mutations in the PEX1 gene are found in approximately $65 \%$ of patients affected with peroxisome biogenesis disorders. Understanding the role of peroxisomes in immunity could be essential to developing new treatments for peroxisome biogenesis disorders. The cure to Zellweger syndrome or neonatal adrenoleukodystrophy may be in the connection between the immune system and peroxisomes. Although much more research is necessary, it could be possible that this research will have an impact on medicine.

\section{CONCLUSION}

This research uncovered evidence that peroxisomes are important to the innate immune response. It was shown that PEX1 null fibroblasts are unable to undergo the c-Fos pathway following infection, although PEX7 null fibroblasts are able. This shows that peroxisomes play a role in immune signalling. In addition, a survivorship curve showed that PEX1 null $D$. melanogaster have a reduced survival rate post-infection when compared to control wild-type $D$. melanogaster. This demonstrates that without peroxisomes, the entire innate immune system is greatly inhibited. Finally, infected S2R+ D. melanogaster macrophages showed that PEX1 deficient cells were unable to undergo the NF-KB immune response. Also, adding palmitate to the macrophage showed that greater concentrations of fatty acids can inhibit the immune response. Overall, this research shows that peroxisomes are important to several parts of the innate immune system.

\section{ACKNOWLEDGEMENTS}

Thank you to Dr. Francesca Di Cara for designing the methods as well as allowing me to use her lab and equipment over the course of the project. As well, thank you to Dr.
Gabrielle Tompkins and the Dalhousie Integrated Science Program for setting up this project and giving me the opportunity to partake in research.

\section{REFERENCES}

1. Jung J, Zeng $\mathrm{H}$, Horng $\mathrm{T}$. Metabolism as a guiding force for immunity. Nat Cell Biol. 2019;(21):85-93. https://www. nature.com/articles/s41556-018-0217-x

2. Gordon S. Phagocytosis: an immunobiologic process. Immunity.2016; 44(3): 463-475. https://www.cell.com/ immunity/fulltext/S1074-7613(16)30065-6

3. Bautista-Hernandez LA, Gomez-Olivares JL, BuentelloVolante B, Bautista- de Lucio V. Fibroblasts: the unknown sentinels eliciting immune responses against microorganisms. Eur J Microbiol Immunol. 2017;7(3): 151-157. https://www.ncbi.nlm.nih.gov/pmc/articles/ PMC5632742/

4. Cooper GM. The cell: a molecular approach. 2nd ed. Sunderland: Sinauer Associates; c2006. https://www.ncbi. nlm.nih.gov/books/NBK9930/

5. Di Cara F, Sheshachalam A, Braverman NE, Rachubinski RA, Simmonds AJ. Peroxisome-mediated metabolism is required for immune response to microbial infection. Immunity. 2017;47(1):93-106. https://www.ncbi.nlm.nih. gov/pubmed/287235

6. Farré JC, Mahalingham $S$, Subramani S. Peroxisome biogenesis, membrane contact sites, and quality control. EMBO. 2018;20(1):1-25. http://embor.embopress.org/ content/early/2018/12/10/embr.201846864.figures-only

7. Hop H, Arayan L, Huy T, Reyes A, Vu S, Min W, et al. The key role of c-fos for immune regulation and bacterial dissemination in brucella infected macrophage. Front. Cell. Infect. Microbiol. 2018;8(287): 1-16. https://www. ncbi.nlm.nih.gov/pubmed/30186773

8. Zhang Q, Lenardo MJ, Baltimore D. 30 years of NF-KB: a blossoming of relevance to human pathology. Cell. 2017;168(1): 37-57. https://www. cell.com/cell/fulltext/S0092-8674(16)31726-3? returnURL=https\%3A\%2F\%2Flinkinghub.elsevier.

9. Liu T, Zhang L, Joo D, Sun S. NF-KB signalling in inflammation. Signal Transduct Target Ther. 2017;2(17): 17023. https://www.ncbi.nlm.nih.gov/pmc/articles/ PMC5661633/

10. Melo-Cavalcante A, Sousa L, Alencar M, Santos J, Mata A, Paz M, et al. Retinol palmitate and ascorbic acid: role in oncological prevention and therapy. Biomed Pharmacother. 2019;109(1): 1394-1405. https://www.sciencedirect.com/science/article/pii/ S0753332218341325

11. Fernandes IR, Russo FB, Pignatari GC, Evangelinellis MM, Tavolari S, Muotri AR et al. Fibroblast sources: where can we get them? Cytotechnology. 2016;68(2):223-228. https:// www.ncbi.nlm.nih.gov/pmc/articles/PMC4754245/

12. Barber R, Harmer D, Coleman R, Clark B. GAPDH as a 
housekeeping gene; analysis of GAPDH mRNA expression in a panel of 72 human tissues. Physiol Genomics. 2005; 21:389-395. https://www.physiology.org/doi/full/10.1152/ physiolgenomics.00025.2005?url ver=Z39.88-2003\&rfr id=ori:rid:crossref.org\&rfr dat=cr pub\%3dpubmed

13. Mast FD, Li J, Virk MK, Hughes SC, Simmonds AJ, Rachubinski RA. A drosophila model for the Zellweger spectrum of peroxisome biogenesis disorders. Dis Model Mech. 2011;4(5):659-672. https://www.ncbi.nlm.nih.gov/ pmc/articles/PMC3180231/

14. Baron MN, Klinger CM, Rachubinski RA, Simmonds AJ. A systemic cell-based analysis of predicted drosophila peroxisomal proteins. Traffic. 2016;17(5): 536-553. https:// www.ncbi.nlm.nih.gov/pubmed/26865094

15. Riera-Borull M, Cuevas V, Alonso B, Vega M, Joven J, Izquierdo $\mathrm{E}$, et al, Palmitate conditions macrophages for enhanced responses toward inflammatory stimuli via JNK activation. J Immunol. 2017;10(21): 1-12. https://www. ncbi.nlm.nih.gov/pubmed/29061766

16. De Jong AJ, Kloppenburg M, Toes REM, Facsinay AL. Fatty acids, lipid mediators, and T-cell function. Front Immunol. 2014;5:483. https://www.frontiersin.org/articles/10.3389/ fimmu.2014.00483/full

\section{APPENDIX}

\section{Relish}
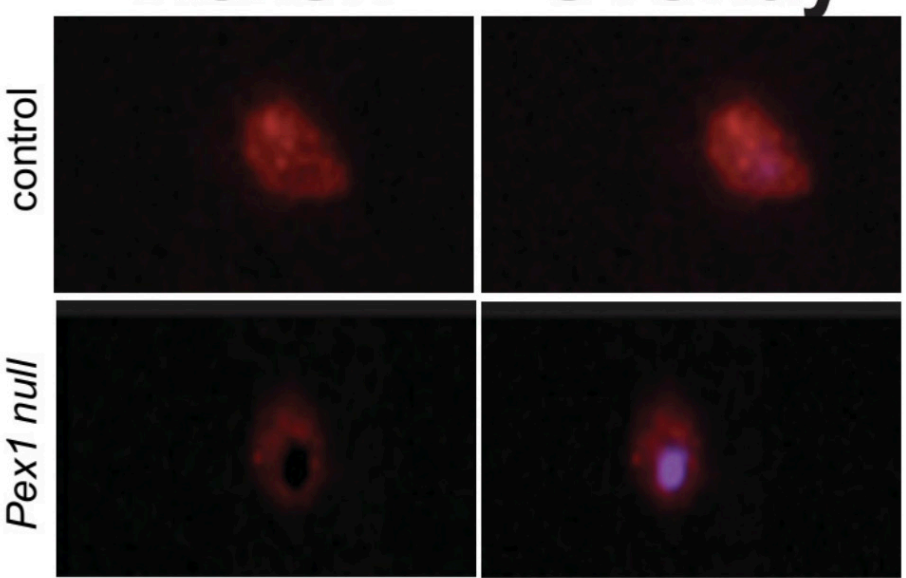

+ E. coli

Figure S1. Immunofluorescence data showing the concentration of a NF- $K B$ in control and 1 null D. melanogaster cells following infection (figure adapted from Di Cara, data not published). 